\title{
A Mediator subunit, MDT-15, integrates regulation of fatty acid metabolism by NHR-49-dependent and -independent pathways in C. elegans
}

\author{
Stefan Taubert, ${ }^{1}$ Marc R. Van Gilst, ${ }^{1,3}$ Malene Hansen, ${ }^{2}$ and Keith R. Yamamoto ${ }^{1,4}$ \\ ${ }^{1}$ Department of Cellular and Molecular Pharmacology and ${ }^{2}$ Department of Biochemistry and Biophysics, University of \\ California, San Francisco, San Francisco, California 94143, USA
}

The Caenorhabditis elegans Nuclear Hormone Receptor NHR-49 coordinates expression of fatty acid (FA) metabolic genes during periods of feeding and in response to fasting. Here we report the identification of MDT-15, a subunit of the C. elegans Mediator complex, as an NHR-49-interacting protein and transcriptional coactivator. Knockdown of $\boldsymbol{m d t} \mathbf{1 5}$ by RNA interference (RNAi) prevented fasting-induced mRNA accumulation of NHR-49 targets in vivo, and fasting-independent expression of other NHR-49 target genes, including two FA- $\Delta$ 9-desaturases (fat-5, fat-7). Interestingly, mdt-15 RNAi affected additional FA-metabolism genes (including the third FA- $\Delta$ 9-desaturase, fat-6) that are regulated independently of NHR-49, suggesting that distinct unidentified regulatory factors also recruit MDT-15 to selectively modulate metabolic gene expression. The deregulation of FA- $\Delta$ 9-desaturases by knockdown of $\mathbf{m d t}-15$ correlated with dramatically decreased levels of unsaturated FAs and multiple deleterious phenotypes (short life span, sterility, uncoordinated locomotion, and morphological defects). Importantly, dietary addition of specific polyunsaturated FAs partially suppressed these pleiotropic phenotypes. Thus, failure to properly govern FA- $\Delta$ 9-desaturation contributed to decreased nematode viability. Our findings imply that a single subunit of the Mediator complex, MDT-15, integrates the activities of several distinct regulatory factors to coordinate metabolic and hormonal regulation of FA metabolism.

[Keywords: Nuclear Hormone Receptor; Mediator; transcriptional coactivator; fatty acid metabolism; $C$. elegans; PUFA]

Supplemental material is available at http://www.genesdev.org.

Received November 28, 2005; revised version accepted March 1, 2006.

Misregulation of energy homeostasis constitutes one of the most prevalent health risks in Western cultures, and can result in disorders such as obesity, diabetes, and metabolic syndrome. The molecular mechanisms and regulatory pathways that govern metabolism of glucose and fat, the principal energy sources in physiological settings, are extremely complex, and remain poorly understood. Nevertheless, processes such as fatty acid (FA) and cholesterol homeostasis are highly conserved, with remarkable similarity, for example, between mammals and invertebrates such as Caenorhabditis elegans (Ashrafi et al. 2003; R.M. McKay et al. 2003). As in mammals, both serotonin signaling (Sze et al. 2000) and insu-

\footnotetext{
${ }^{3}$ Present address: Fred Hutchinson Cancer Research Center, Basic Sciences Division, Seattle, WA 98109-1024, USA.

${ }^{4}$ Corresponding author.

E-MAIL yamamoto@cmp.ucsf.edu; FAX (415) 476-6129.

Article and publication are at http://www.genesdev.org/cgi/doi/10.1101/ gad.1395406
}

lin signaling (Kimura et al. 1997) contribute to proper regulation of fat metabolism in C. elegans. Similarly, members of the Nuclear Hormone Receptor (NHR) family of transcriptional regulators influence fat storage in C. elegans and mammals alike (Desvergne and Wahli 1999; Ashrafi et al. 2003).

NHRs comprise a family of hormone-gated regulatory factors: The ligand-bound receptors associate with cognate hormone response elements to modulate target gene transcription. Specifically, association of small molecules with ligand-binding domains (LBDs) of NHRs modulates interactions of the receptors with coregulatory proteins and with DNA, hence changing the regulatory output (Glass and Rosenfeld 2000). Thus, LBDs serve as powerful molecular switches that enable the NHRs to edit physiological programs in response to hormonal or environmental stimuli. However, despite detailed biochemical analysis of several ligand-controlled LBD:coregulator interactions, and the well-characterized role of many NHRs in endocrine signaling, the specific 
physiological actions of many NHR:coregulator complexes are just beginning to emerge.

The human genome encodes 48 NHRs, which govern many distinct developmental and physiological processes. Human NHRs include steroid hormone receptors-for example, Glucocorticoid Receptor (GR) - and nonsteroid hormone receptors such as the PeroxisomeProliferator-Activated Receptors (PPARs) and Hepatocyte Nuclear Factor $4 \alpha$ (HNF4 $\alpha$ ) (Evans 1988). Both steroid and nonsteroid NHRs, including the PPARs $(\operatorname{PPAR} \alpha, \operatorname{PPAR} \beta / \delta$, and $\operatorname{PPAR} \gamma)$, regulate fat, glucose, and cholesterol homeostasis (Desvergne and Wahli 1999; Handschin and Meyer 2005). In particular, PPAR $\alpha$ is a central regulator of FA metabolism whose activation results in reduced serum levels of triacylglycerides, free FAs, and cholesterol. Accordingly, synthetic PPAR $\alpha$ ligands are efficient lipid-lowering drugs used for treatment of diabetes (Desvergne and Wahli 1999).

Although the C. elegans genome encodes 284 NHRs, none exhibits significant sequence similarity to the PPARs; instead, many appear to be derivatives of an ancient HNF4 $\alpha$-like NHR (Sluder and Maina 2001; Van Gilst et al. 2002). However, one of these, NHR-49, functionally resembles human PPAR $\alpha$ : Like PPAR $\alpha$, NHR-49 is necessary for expression of genes involved in FA- $\beta$ oxidation, lipid binding, and FA- $\Delta$ 9-desaturation (Nakamura et al. 2004; Van Gilst et al. 2005a,b). Consistent with this functional resemblance, reduced levels of nhr49 result in increased body fat (Ashrafi et al. 2003; Van Gilst et al. 2005a), an elevated ratio of saturated to monounsaturated FAs, an impaired response to short-term fasting, and shortened life span (Van Gilst et al. 2005a,b). This panel of phenotypes resembles the characteristics of mice lacking functional PPAR $\alpha$ (Lee et al. 1995; Vega et al. 2000), suggesting that NHR-49 and PPAR $\alpha$ perform similar regulatory roles in vivo.

HNF $4 \alpha$ and PPAR $\alpha$ engage a multitude of transcriptional coregulators, including the corepressor NCor (Dowell et al. 1999), and coactivators such as p300/CBP (Dowell et al. 1997), the PPAR $\gamma$-coactivators PGC-1 $\alpha$ and PGC-1 $\beta$ (Vega et al. 2000), and the Mediator subunit MED1/TRAP220/DRIP205/PBP/CRSP200 (subsequently named MED1) (Bourbon et al. 2004; Malik et al. 2004). In addition, med1 $1^{-1-}$ mouse embryonic fibroblasts fail to properly express many PPAR $\gamma$ target genes, and are incapable of undergoing proper adipocyte differentiation, a PPAR $\gamma$-dependent cell fate decision (Ge et al. 2002). Similarly, PGC-1 proteins stimulate mitochondrial proliferation, oxidative phosphorylation, adaptive thermogenesis, and the fasting response in the liver (Puigserver and Spiegelman 2003; Lin et al. 2005). PGC-1 binds distinct regulatory factors to mediate these metabolic and biogenic responses, including $\operatorname{PPAR} \alpha, \operatorname{PPAR} \gamma$, and HNF4 $\alpha$.

The C. elegans genome appears to lack PGC-1-related proteins (Knutti et al. 2000); in addition, the conservation between mammalian MED1 and its C. elegans ortholog, MDT-1.1/SOP-3, is weak (Blazek et al. 2005). Thus, we hypothesized that distinct C. elegans coregulators might confer physiological effects similar to those coordinated by PGC-1 and MED1. In this study, we sought to identify such potential components of NHR-49 regulatory complexes, and to investigate their overall physiological role in C. elegans energy homeostasis.

\section{Results}

Specific interaction between NHR-49 and MDT-15

To identify potential coregulators of NHR-49, we performed a yeast two-hybrid screen using a GAL4 DNAbinding domain (DBD) NHR-49-LBD fusion protein to probe a mixed-stage C. elegans GAL4-activation domain (AD)-cDNA library for interacting proteins, and identified six clones corresponding to full-length MDT-15 (R12B2.5). Using a GAL-4-driven LacZ-reporter, we estimated that binding of this MDT-15 fusion protein to the GAL4-DBD-NHR-49-LBD was at least 200-fold stronger than association with the GAL4-DBD alone (Fig. 1A).

The C. elegans genome encodes 284 NHRs. To test the possibility that some of these NHRs recruit the same coregulator, we assessed the binding of MDT-15 to 11 GAL4-DBD-NHR-LBD fusions. Five of these LBDs (NHR-3, NHR-14, NHR-31, NHR-35, NHR-64) display relatively strong similarity to the NHR-49-LBD (Sluder and Maina 2001; Van Gilst et al. 2002), and reduced expression of the other six NHRs (NHR-68, NHR-88, NHR-137, NHR-140, NHR-178, and DAF-12) results in defective fat storage (Ashrafi et al. 2003). Of these additional LBDs, only the NHR-64-LBD interacted with MDT-15 in the yeast two-hybrid system (Fig. 1A). All LBD fusions were expressed at similar levels; moreover, the LBDs of NHR-3, NHR-14, NHR-31, NHR-35, and NHR-64 homodimerized (data not shown), and the DAF12-LBD interacted with the corepressor DIN-1S (Ludewig et al. 2004), suggesting that these LBDs assume their functional conformations. Taken together, these data imply that association of NHR-49 (and NHR-64) with MDT-15 is selective and may be biologically significant.

The $m d t-15$ gene is predicted to produce two MDT-15 isoforms differing in only three amino acids. The MDT15 proteins exhibit sequence similarity to yeast MED15/ GAL11 (subsequently named GAL11) (Bourbon et al. 2004) and human MED15/ARC105/TIG-1/PCQAP proteins (subsequently named MED15) (Bourbon et al. 2004). GAL11 and MED15 proteins are subunits of the yeast and human Mediator complexes, respectively, multisubunit protein complexes that act as transcriptional coregulators for regulatory factors (Blazek et al. 2005; Malik and Roeder 2005).

MDT-15 and related proteins contain an $\mathrm{N}$-terminal GACKIX domain, which is related to the KIX domain present in the transcriptional coregulators p300/CBP (Novatchkova and Eisenhaber 2004); within these proteins, the KIX domain constitutes a functional target for numerous regulatory factors. Thus, we suspected that the GACKIX domain of MDT-15 might mediate the interaction with NHR-49 and other C. elegans regulatory factors. To address this possibility, we performed a yeast 
A

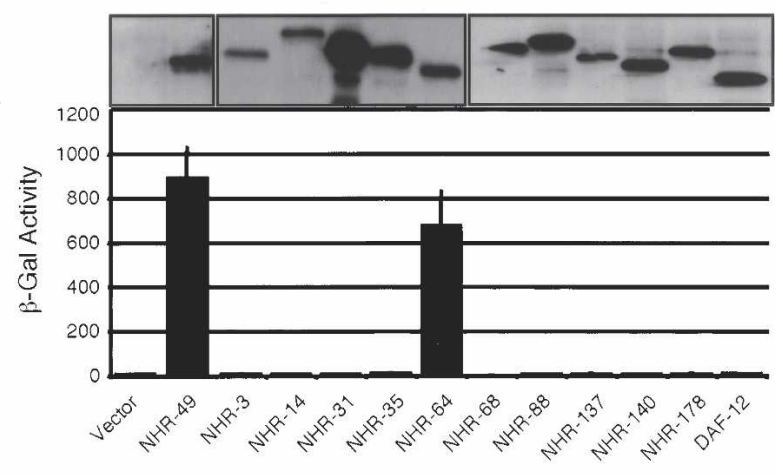

B

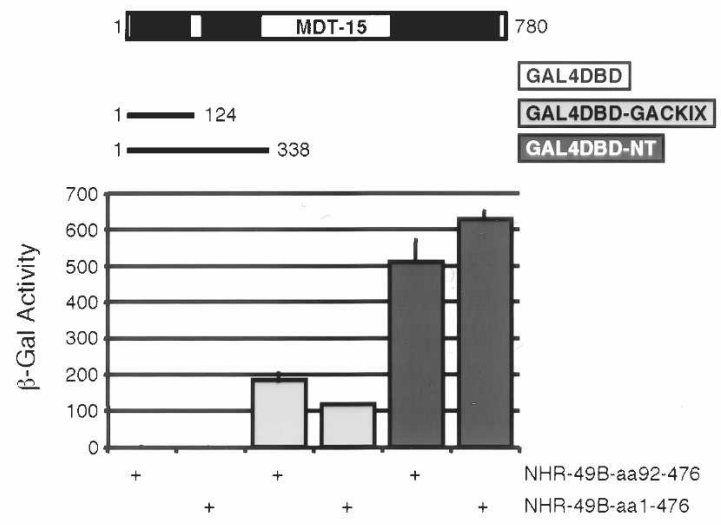

C

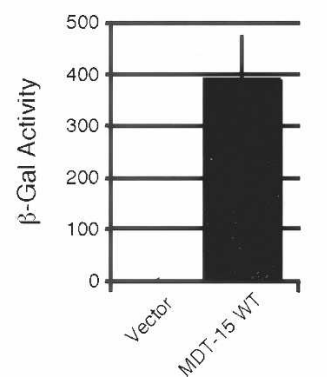

Figure 1. MDT-15 specifically interacts with the NHR-49-LBD and confers activation capability in yeast. $(A)$ Estimates of the relative interaction strength of $C$. elegans NHR-LBDs with MDT-15 using the yeast two-hybrid system and $\beta$-galactosidase reporter assays. Values indicate average interaction strength from quantification of six independent transformants for each plasmid combination; error bars represent SEM. The top panel shows protein expression of GAL4-DBD-NHR-LBD fusions as determined by Western blot. $(B)$ Estimates of the relative interaction strength of two MDT-15 proteins with two independent NHR-49 clones; the schematic on top depicts MDT-15 fusion proteins. Values indicate average interaction strength from quantification of eight independent transformants for each plasmid combination; error bars represent SEM. $(C)$ Estimate of the activation capability of the GAL4-DBD-MDT-15 fusion protein using $\beta$-galactosidase activity assay (average of six independent transformants for each plasmid); error bars represent SEM.

two-hybrid screen using the MDT-15-GACKIX domain as bait. We identified two distinct clones encoding NHR49, both encompassing the whole LBD. Using the GAL4- driven LacZ reporter, we estimated that binding of both NHR-49 clones to two distinct GAL4-DBD-MDT-15 fusion proteins including the GACKIX domain was at least 100 -fold stronger than association with the GAL4-DBD alone (Fig. 1B). Therefore, the MDT-15-GACKIX domain likely represents an interaction target for the NHR-49LBD.

In view of the coregulatory function of mammalian MED15 and the intrinsic activity of yeast GAL11, we tested whether C. elegans MDT-15 similarly possesses activating capability. To this end, we fused the fulllength ORF of MDT-15 to the GAL4-DBD, and assayed the capacity of this fusion protein to activate a GAL4driven LacZ reporter. GAL4-DBD-MDT-15, but not the empty vector, strongly induced $\beta$-galactosidase activity (Fig. 1C) suggesting that, at least in yeast, MDT-15 can act as a transcriptional coactivator when recruited to DNA.

The mdt-15 promoter drives intestinal and neuronal expression in C. elegans

To determine the temporal expression profile of C. elegans mdt-15, we used quantitative real-time PCR analysis (qRT-PCR) to assess mdt-15 mRNA levels in vivo throughout development. These experiments revealed that mdt-15 mRNA is expressed at similar levels in embryos, at all four larval stages, and in adults (Fig. 2A). To corroborate these results, and to assess the spatial expression pattern of $m d t-15$ expression, we used a transgenic $C$. elegans strain carrying a transcriptional fusion of 1163 nucleotides (nt) upstream of the mdt-15 ORF to GFP (BC11928) (S.J. McKay et al. 2003). Consistent with the qRT-PCR results, we detected GFP fluorescence at all developmental stages (Fig. 2; Supplementary Fig. S1). Interestingly, expression appeared to localize to the intestine (Fig. 2B) and to several head neurons (Fig. 2C). By comparison, NHR-49 is expressed in the intestine, pharynx, hypodermis, and body wall muscle (Van Gilst et al. 2005a). Although this expression pattern suggests that NHR-49 may function in many tissues, it is striking that NHR-49 and MDT-15 are coexpressed in the intestine, which is the principal organ of fat storage and metabolism in C. elegans. The partial overlap in the expression patterns of $m d t-15$ and $n h r-49$ is consistent with the notion that they are shared components of a regulatory network in a physiological setting.

MDT-15 is necessary for mRNA accumulation of NHR-49 targets in vivo

A C. elegans strain carrying a deletion in the nhr-49 gene, nhr-49(nr2041), exhibits impaired accumulation of mRNA from specific FA-metabolism genes in response to fasting (Van Gilst et al. 2005b). Given that MDT-15 binds NHR-49, we hypothesized that it participates in the fasting response, thus acting as an NHR-49 coregulator in vivo. To test this hypothesis, we knocked down 
Taubert et al.

A

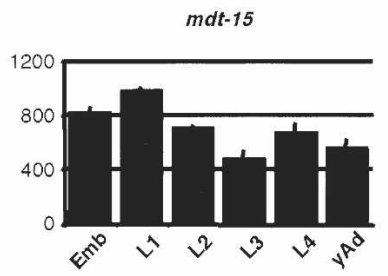

B

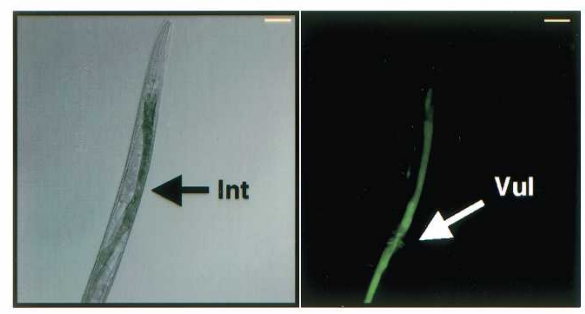

C

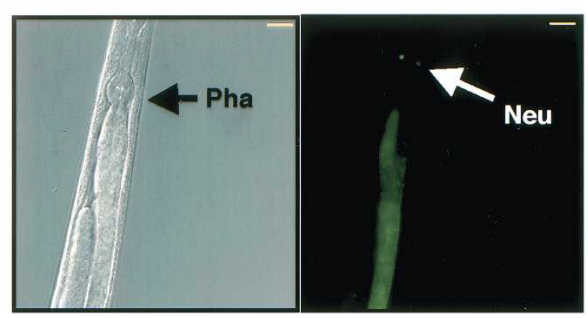

Figure 2. Analysis of $m d t-15$ expression. (A) Relative levels of mdt-15 mRNA (normalized to ama-1 mRNA levels) in embryonic, larval, and adult N2 worms were determined by qRT-PCR. The bars represent averages of three independent experiments; error bars represent SEM. $(B, C)$ Fluorescence and DIC micrographs of $\mathrm{BC} 11928$ worms at the early adult stage. The promoter of $m d t-15$ drives expression in the intestine $(B)$, and in head neurons $(C)$. Bars: $B, 129 \mu \mathrm{m} ; C, 49 \mu \mathrm{m}$. (Pha) Pharynx; (Neu) head neurons; (Vul) vulva; (Int) intestine.

endogenous NHR-49 or MDT-15 in wild-type worms using feeding RNA-interference (RNAi) (for review, see Wang and Barr 2005), and then monitored by qRT-PCR the mRNA levels of fasting-responsive FA-metabolism genes at the L4 stage. Importantly, qRT-PCR and immunoblot analysis confirmed that $m d t-15 \mathrm{RNAi}$, but not $n h r-49$ or mdt-6 RNAi, significantly reduced MDT-15 mRNA and protein levels in vivo relative to control(RNAi) worms (Supplementary Fig. S2).

Consistent with previous findings (Van Gilst et al. 2005b), mRNA abundance of four NHR-49 target genes, the acyl-CoA synthetases (ACS) acs-2 and acs-11, the malate synthase/isocitrate lyase gei-7, and the 3-hydroxyacyl-CoA dehydrogenase hacd-1 (Fig. 3A), but not of the $n h r-23$ gene (Fig. 3C) were strongly up-regulated after $8 \mathrm{~h}$ of fasting in control(RNAi) worms, but not in nhr-49(RNAi) worms. Similarly, mdt-15(RNAi) worms failed to accumulate mRNA of these NHR-49 target genes (Fig. 3A). Unlike in nhr-49(nr2041) animals, mRNA abundance of these genes after $n h r-49$ or mdt-15 RNAi was unaffected in the basal (fed) state; possibly, residual NHR-49 and MDT-15 proteins are sufficient for basal transcription. In any case, our data suggest that MDT-15 is necessary for fasting-induced transcription of NHR-49 target genes in vivo.

In addition to inducing gene expression as part of the fasting response, NHR-49 is required for expression of several FA-metabolism genes in the fed state, such as the FA- $\Delta$ 9-desaturases fat-5 and fat-7, the lipid-binding protein (LBP) $1 b p-8$, and the carnitine-palmitoyl-transferase (CPT) cpt-5 (Van Gilst et al. 2005a). Strikingly, both mdt15 RNAi and nhr-49 RNAi, but not control RNAi, drastically reduced expression of these genes, regardless of nutrient state (Fig. 3B). These results imply that NHR-49 and MDT-15 also collaborate to control an additional sector of FA metabolism besides up-regulating a subset of genes as part of the fasting response.

Given that $m d t-15$ RNAi reduced mRNA levels of several NHR-49 targets, it seemed plausible that MDT-15 could regulate transcription of nhr-49 itself. However, control(RNAi) and mdt-15(RNAi) worms exhibited similar levels of $n h r-49$ mRNA, regardless of nutritional state (Fig. 3C). We conclude that alteration of mRNA levels of NHR-49 targets after exposure to mdt-15 RNAi is likely a direct consequence of reduced MDT-15 levels.

\section{MDT-15 regulates NHR-49-independent FA-metabolism genes}

Van Gilst et al. (2005b) have recently reported that shortterm fasting of N2 L4 worms alters mRNA abundance of 23 genes involved in FA metabolism. Only four of those genes require nhr-49 for appropriate response to food withdrawal. We wished to determine whether the NHR49-independent fasting-responsive genes are also MDT15 targets and thus measured relative mRNA levels of a subset of those genes after individual RNAi treatments. Indeed, mdt-15 RNAi, but not $n h r-49$ RNAi, reproducibly altered mRNA levels of five fasting-responsive genes more than fourfold in L4 larvae (Fig. 3D). One of these genes, acdh-2, is predicted to encode a short-chain acylCoA-dehydrogenase (SCADH), whose expression is reduced by fasting. mdt-15 RNAi severely impaired mRNA accumulation of acdh-2 in the fed state $(\sim 10$ fold); in the fasted state, mRNA levels were further reduced, but only approximately twofold. For a second gene, $1 b p-1$, mRNA abundance was similar in fed control(RNAi), nhr-49(RNAi), and mdt-15(RNAi) animals, whereas fasting-induced mRNA accumulation was compromised after mdt-15 RNAi. In addition, regardless of feeding state, mdt-15(RNAi) worms exhibited severely diminished mRNA levels of fat-6, the FA- $\Delta$ 9-desaturase only marginally affected in $n h r-49(n r 2041)$ worms (Van Gilst et al. 2005a). Furthermore, mdt-15(RNAi) worms exhibited an approximately fourfold reduction of mRNA levels of the FA- $\Delta 12$-desaturase fat-2 in both fed and fasted conditions. Finally, we identified one gene, $c p t-3$, whose fasting-induced expression was further stimulated by mdt-15 RNAi.

Taken together, our data suggest that MDT-15 collaborates with multiple regulatory factors to precisely 


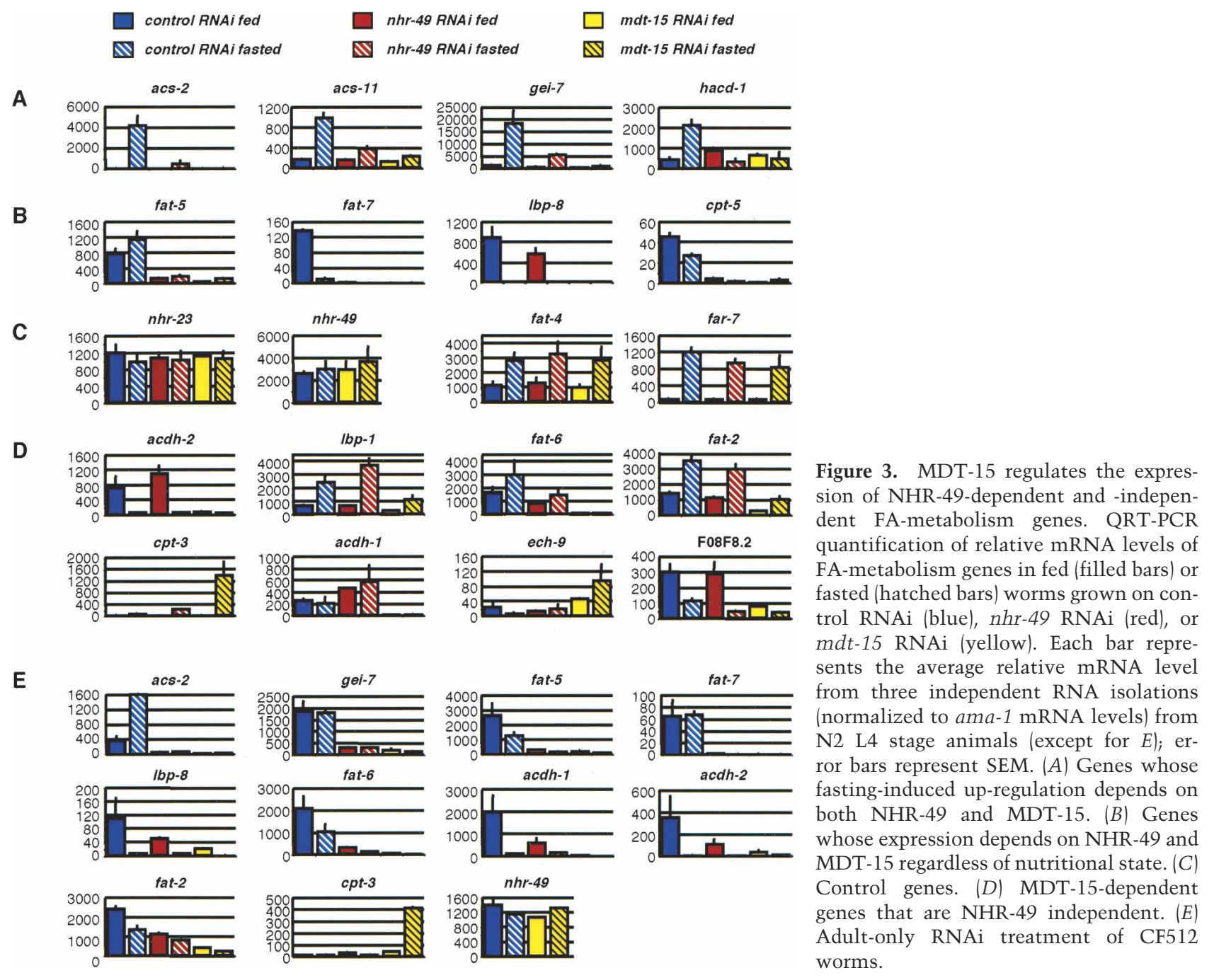

govern expression of a set of FA-metabolism genes in response to short-term fasting, as well as in the fed state. However, we note that MDT-15 was not required for fasting-stimulated mRNA accumulation of other genes, such as the FA/retinol-binding-protein far-7 (Garofalo et al. 2003), and the FA- $\Delta 5$-desaturase fat-4 (Fig. 3C). Thus, we speculate that unidentified regulatory complexes that do not require NHR-49 or MDT-15 activities modulate expression of this sector of the fasting response.

\section{MDT-15 regulates selected fasting-independent FA-metabolism genes}

Steady-state mRNA levels of most FA-metabolism genes are mostly refractory to short-term fasting (Van Gilst et al. 2005b). Since MDT-15 was necessary for basal expression of a subset of those genes, we expanded our mRNA survey to include 96 FA-metabolism genes in worms continuously fed control RNAi or mdt-15 RNAi (Table 1; Supplementary Table S1). We found five additional genes that reproducibly exhibited more than fourfold alterations of mRNA levels in mdt-15(RNAi) worms (Fig.
3D; Supplementary Table S1). These included another SCADH (acdh-1), one enoyl-CoA hydratase (ech-9), and three acyl-CoA oxidases (F08A8.2-4). Another 13 FA-metabolism genes were deregulated two- to fourfold; the rest did not show altered expression levels after $m d t-15$ RNAi (Supplementary Table S1). Overall, mdt-15(RNAi) worms clearly exhibited more severe defects in FA-metabolic gene expression than nhr-49(nr2041) or nhr49(RNAi) animals.

\section{MDT-15 regulates FA-metabolism genes in adult worms}

Impaired gene expression at the L4 stage as described above could represent secondary effects of $m d t-15$ RNAi during larval development (see below). To address this concern, we analyzed the consequence of $m d t-15$ knockdown on gene expression in adults, using a conditionally sterile strain (CF512) (Garigan et al. 2002). Analysis of mRNA abundance revealed defects in gene expression similar to those in RNAi-treated L4 larvae: Basal and fasting-induced levels of acs-2 transcripts were reduced 
Table 1. Summary of gene expression changes in fed mdt-15(RNAi) worms relative to fed control(RNAi) worms

\begin{tabular}{|c|c|c|c|c|c|c|c|}
\hline & Total transcripts & $\mathrm{Up}>4 \times$ & Up 2-4x & Between 2 and $-2 x$ & Down -2 to $-4 \times$ & Down $>4 \times$ & Total deregulated \\
\hline Fat metabolism & 96 & $1(1 \%)$ & $8(8 \%)$ & $72(75 \%)$ & $6(6 \%)$ & $9(9 \%)$ & $24(25 \%)$ \\
\hline Glucose metabolism & 43 & $0(0 \%)$ & $3(7 \%)$ & $34(79 \%)$ & $6(13.9 \%)$ & $0(0 \%)$ & $9(21 \%)$ \\
\hline DAF-12 & 30 & $0(0 \%)$ & $1(3 \%)$ & $27(90 \%)$ & $0(0 \%)$ & $2(7 \%)$ & $3(10 \%)$ \\
\hline Total & 169 & $1(1 \%)$ & $12(7 \%)$ & $133(79 \%)$ & $12(7 \%)$ & $11(7 \%)$ & \\
\hline
\end{tabular}

For further details, see text.

after $m d t-15$ and $n h r-49$ RNAi, and basal levels of NHR49 targets gei-7, fat-5, fat-7, and $1 b p-8$ and of non-NHR49 targets fat-6, acdh-1, acdh-2, fat-2, and cpt-3 were deregulated after mdt-15(RNAi), whereas mRNA levels of $n h r-23$ and $n h r-49$ were not altered (Fig. 3E; data not shown); note that many genes that respond to fasting in L4s are not responsive in adults (Van Gilst et al. 2005b). In support of these results, most animals exposed to $m d t-15$ RNAi as early as the L1 stage exhibited pleiotropic phenotypes only at day 1 of adulthood; thus, we observed transcriptional changes well before the major phenotypic changes were evident (see below). We conclude that the observed defects in FA-metabolism gene expression most likely result directly from reduced levels of $m d t-15$, and do not reflect impaired larval development.

\section{Selective action of MDT-15}

How selective are the actions of MDT-15? In mammals, the expression of certain glucose-metabolism genes requires coregulators such as PGC-1 that modulate fat metabolism (Nakamura et al. 2004). Thus, we surveyed mRNA abundance of 43 transcripts from 37 genes predicted to be involved in glycolysis, gluconeogenesis, the Krebs cycle, and sugar transport (Table 1; Supplementary Table S2). Levels of nine of these mRNAs were reproducibly altered after mdt-15 RNAi; however, no transcript was deregulated more than fourfold. Furthermore, only two of 30 transcripts regulated by the NHR DAF-12 (Shostak et al. 2004), which governs a complex response to nutrient deprivation, exhibited more than fourfold deregulation in fed mdt-15(RNAi) animals (Table 1; Supplementary Table S3). Overall, whereas only two of 73 glucose metabolism and DAF-12 target genes were modulated more than fourfold, 10 of 96 fat-metabolism genes were deregulated to this extent in mdt-15(RNAi) worms (Table 1); indeed, several FA-metabolism genes were deregulated $>100$-fold. Taken together, these data suggest that regulation by MDT-15 may be rather selective, and that it does not globally influence transcription (Table 1). Furthermore, the data also demonstrate that most (nine of 10 more than fourfold affected genes) of the affected genes are down-regulated after mdt-15 RNAi, implying that activation may be a predominant function for MDT-15 in vivo.

As MDT-15 is predicted to reside in the Mediator complex, deregulation of FA-metabolism genes could, in principle, occur in response to knockdown of any Mediator subunit-that is, as result of a general loss of Me- diator activity. To address this possibility, we determined relative mRNA levels of select MDT-15 targets in $m d t$-15(RNAi) worms and mdt-6(RNAi) worms. We choose MDT-6 because it is required for stage- and genespecific transcription in C. elegans (Kwon and Lee 2001). mdt-6 RNAi resulted in a larval arrest phenotype (data not shown) similar to that described for a null mutation in the mdt-6 gene (1et-425) (Kwon and Lee 2001). Interestingly, despite this developmental arrest, mdt-6(RNAi) worms exhibited only a weak reduction of fat-5 and fat- 6 mRNA levels, whereas reduction of mRNA levels of fat-7 and $1 b p-8$ was more substantial, albeit weaker than in $m d t$-15(RNAi) worms (Supplementary Fig. S2). Thus, it appears that the role of MDT-15 in regulation of fat metabolic genes is rather specific, and reduced mRNA levels in mdt-15(RNAi) worms are not the results of general loss of Mediator activity.

\section{MDT-15 affects fat storage}

nhr-49(nr2041) worms exhibit a high fat phenotype due to severe defects in expression of the FA- $\beta$-oxidation genes acs-2, acs-11, cpt-5, and hacd-1 (Van Gilst et al. 2005a). As mdt-15(RNAi) also caused reduced mRNA levels of these and other FA- $\beta$-oxidation genes, we examined the effects of mdt-15 RNAi on fat storage, using the lipophilic vital dye Nile Red (Ashrafi et al. 2003; R.M. McKay et al. 2003). Indeed, the Nile Red staining pattern (Fig. 4) indicated that fat distribution was much more diffuse in mdt-15(RNAi) worms, compared with the accumulation of fat droplets in control(RNAi) worms. Although the diffuse nature of the Nile Red staining in mdt-15 RNAi animals did not allow precise comparison of lipid levels, we conclude that fat storage is altered as a consequence of reduced $m d t-15$ expression.

\section{$M D T-15$ is required for maintenance of regular FA-composition}

The strong down-regulation of fat-5, fat-6, and fat-7 in mdt-15(RNAi) animals is distinct from the expression profile found in nhr-49(nr2041) and nhr-49(RNAi) worms, in which fat-6 expression is only slightly impaired (Fig. 3). These genes encode FA- $\Delta$ 9-desaturases that metabolize palmitic $(\mathrm{C} 16: 0$; fat-5) and stearic acid (C18:0; fat-6 and fat-7) to produce palmitoleic (C16:1n7) and oleic acid (C18:1n9), respectively (Fig. 5A). Thus, FAT-6 and FAT-7 produce monounsaturated FA (MUFA) precursors for the synthesis of polyunsaturated FAs 
control RNAi

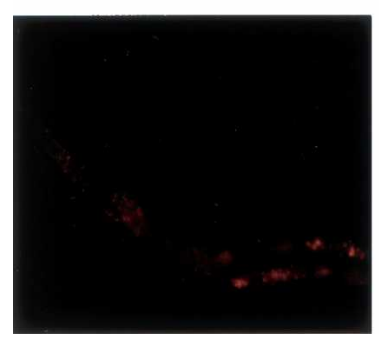

mdt-15 RNAi

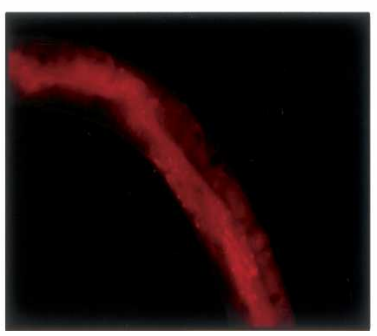

$m d t-15 \mathrm{RNAi}+\mathrm{ETA} / \mathrm{EPA}$

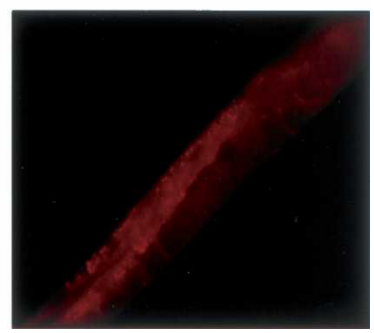

Figure 4. MDT-15 regulates fat storage. Fluorescence micrographs (200x magnification) reveal altered fat storage in $m d t$ 15(RNAi) versus control(RNAi) animals, as detected by staining with the vital dye Nile Red. Dietary FAs (100 $\mu$ M C20:5 and $100 \mu \mathrm{M}$ C20:3n6) partially suppress the defect.
(PUFAs) (Watts and Browse 2002). Notably, however, neither nhr-49 nor fat-7 knockdown results in altered PUFA levels in vivo; instead, these animals exhibit an altered ratio of $\mathrm{C} 18: 0 / \mathrm{C} 18: \ln 9$ ratio, suggesting that this conversion is the principal function of fat-7, whereas fat-6 is responsible for production of PUFA precursors (Van Gilst et al. 2005a).

Given that all three FA- $\Delta$ 9-desaturases are strongly down-regulated in mdt-15(RNAi) worms, and that these appear to be the only FA- $\Delta$ 9-desaturases in the $C$. elegans genome, we expected mdt-15(RNAi) worms to exhibit both an altered $\mathrm{C} 18: 0 / \mathrm{C} 18: \ln 9$ ratio as well as reduced amounts of MUFAs and PUFAs. To address this hypothesis, we extracted FAs from RNAi-treated animals, and analyzed the extracts by gas chromatography/mass spectrometry (GC/MS). nhr-49(RNAi) worms exhibited a C18:0/C18:1n9 ratio of $2.2 \pm 0.2$ (Fig. $5 B$ ), similar to that reported previously (Van Gilst et al. 2005a). In contrast, mdt-15(RNAi) worms displayed a strikingly distinct FA profile (Fig. $5 \mathrm{C}$ ): The absolute amounts of saturated FAs (C16:0 and C18:0) were increased at the expense of all MUFAs and PUFAs except C16:1n7, which was unexpectedly elevated. Consistent with the gene expression data, the levels of vaccenic acid (C18:1n7), linoleic acid (C18:2n6), di-homo- $\gamma$-linolenic acid (C20:3n6), omega-3 arachidonic acid (C20:4n3), arachidonic acid (C20:4n6), and eicosapentaenoic acid (C20:5) were markedly reduced. The levels of $\mathrm{C} 18: \ln 9$ were also reduced, albeit to a lesser extent. These changes resulted in a C18:0/ C18:1n9 ratio of $4.8 \pm 0.8$, substantially higher than the ratio of these components in $n h r-49$ (RNAi) worms $[2.2 \pm 0.2$ (Fig. $5 \mathrm{~B}$ ); note that the ratio is $\sim 4.3$ in $n h r-$ 49(nr2041) animals (Van Gilst et al. 2005a)]. Taken together, these data show that the severe down-regulation of all three FA- $\Delta$ 9-desaturases in $m d t$-15(RNAi) animals is reflected in a dramatic reduction of MUFA and PUFA abundance, suggesting that MDT-15 centrally governs FA- $\Delta$ 9-desaturation in $C$. elegans in vivo.

\section{MDT-15 is critical for normal life span}

The decreased expression of fat-5 and fat-7 in nhr49(nr2041) and nhr-49(RNAi) worms, as well as the reduction of fat-7 levels by RNAi, cause an altered C18:0/ C18:1n9 ratio, and are accompanied by a shortened life span (Van Gilst et al. 2005a). In fact, the highest C18:0/ C18:1n9 ratio [ 8 in fat-7(RNAi) animals] correlates with the shortest life span, whereas the slight increase of the C18:0/C18:1n9 ratio in $n h r-49(R N A i)$ worms correlates with a mild reduction of life span (Van Gilst et al. 2005a). As mdt-15 RNAi strongly increased the C18:0/ C18:1n9 ratio, we suspected that these animals might be short-lived. Indeed, mdt-15 RNAi reduced the adult life span of CF512 worms to a mean of $11.0 \pm 0.1 \mathrm{~d}$, whereas nhr-49 RNAi shortened life span to only $12.8 \pm 0.2 \mathrm{~d}$ (control RNAi, $16.6 \pm 0.2$ d) (Fig. 6A); similar results were obtained with $\mathrm{N} 2$ worms (data not shown). Together, these data suggest that normal expression of MDT-15 is important for maintenance of normal life span, perhaps due to its regulation of the FA- $\Delta$ 9-desaturases.

A

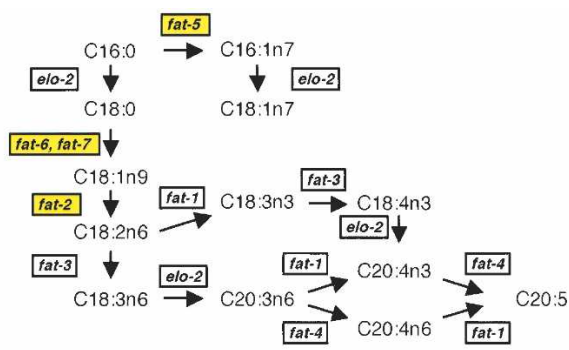

B

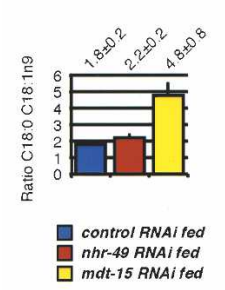

C

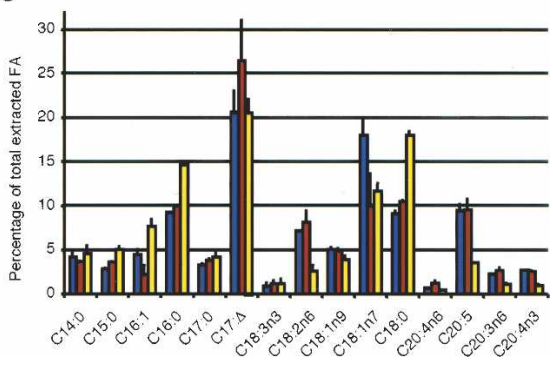

Figure 5. MDT-15 governs PUFA levels and maintains the C18:0/C18:1n9 ratio. (A) The FA-desaturation/elongation pathway of C. elegans (Watts and Browse 2002). Genes highlighted in yellow are down-regulated in mdt-15(RNAi) worms (Fig. 3). (B) C18:0/C18:1n9 ratio, calculated from the data shown in $C$; values are indicated at the top. (C) Relative abundance of FAs, expressed as a fraction of total FA, was determined by GC/MS from N2 L4 worms grown on either control RNAi (blue), nhr-49 RNAi (red), or mdt-15 RNAi (yellow). Bars represent the average relative FA abundance from three independent experiments; error bars represent SEM. 
Figure 6. MDT-15 is necessary for normal life span and animal health. (A) RNAi treatment of CF512 worms was initiated at distinct stages to compare the effect of $n h r-49$ RNAi (red) and mdt-15 RNAi (yellow) on whole-life (WL) life span with the effect of RNAi only during adulthood (adult-only, AO). Mean life spans (measured in days after reaching adulthood) were $16.6 \pm 0.2 \mathrm{~d}$ for control(RNAi) worms (blue; $n=64$ ), $12.8 \pm 0.2 \mathrm{~d}$ for $n h r-49$ AO RNAi (red; $n=42), 11.8 \pm 0.1$ $\mathrm{d}$ for $n h r-49$ WL RNAi (red dotted; $n=50$ ), $11.0 \pm 0.1 \mathrm{~d}$ for $m d t-15$ AO RNAi (yellow; $n=86$ ), and $7.9 \pm 0.1 \mathrm{~d}$ for mdt-15 WL RNAi (yellow dotted; $n=76$ ). (B) DIC micrographs (200× magnification) of N2 worms fed control RNAi or mdt-15 RNAi; mdt-15(RNAi) worms contained vacuoles (arrows) in the intestine and vulva (Vul) area. (Right panel) Dietary FAs (100 $\mu \mathrm{M}$ C20:5 and $100 \mu \mathrm{M} \mathrm{C} 20: 3 \mathrm{n} 6)$ partially suppress these defects. (C) The WL life span of CF512 worms on control RNAi (blue) and mdt-15 RNAi (yellow) was determined on plates containing $\mathrm{H}_{2} \mathrm{O}$ or a combination of PUFAs (100 $\mu \mathrm{M}$ C20:5 and $100 \mu \mathrm{M}$ C20:3n6), respectively. Mean life spans were $20.9 \pm 0.5 \mathrm{~d}$ for control(RNAi) worms (blue; $n=43$ ), $21.9 \pm 0.3 \mathrm{~d}$ for control(RNAi) on PUFAs (blue dotted; $n=40$ ), $9.0 \pm 0.1 \mathrm{~d}$ for mdt15(RNAi) worms (yellow; $n=59$ ), and $10.5 \pm 0.1 \mathrm{~d}$ for mdt-15(RNAi) worms on PUFAs (yellow dotted; $n=71$ ).

\section{MDT-15 is necessary during development and adulthood}

In addition to the short adult life-span phenotype described above, previous studies have noted that mdt15(RNAi) worms appear sick and sluggish and show defects in larval and adult development and behavior (Kamath et al. 2003; Rual et al. 2004). Consistent with these reports, we observed partial larval arrest (data not shown) and an even shorter life span when CF512 eggs-not young adults-were plated on mdt-15 RNAi (Fig. 6A). These observations suggest that mdt-15 expression is critical for both larval and adult development and behavior.

When N2 worms were exposed to mdt-15 RNAi beginning at the L1 stage, almost all developed into adults, but exhibited Unc phenotypes from day 1 of adulthood (data not shown). Unlike control(RNAi) animals, most of these $m d t$-15(RNAi) adults failed to lay eggs; eggs that were laid usually did not hatch, and any hatched worms arrested at the L1 stage. Furthermore, we noted deterioration of the germline and intestine, and onset of vacuole formation throughout the body (Fig. 6B), followed by premature death. Interestingly, whereas nhr-49(nr2041) mutants and nhr-49(RNAi) animals are short-lived, neither larval nor adult development, fertility, nor motility is severely impaired in those worms (Van Gilst et al. 2005a). Thus, the defects in mdt-15(RNAi) worms may

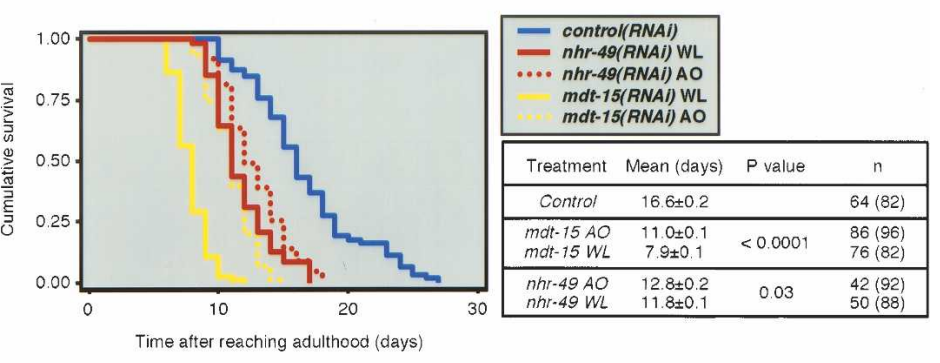

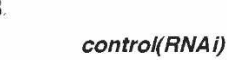

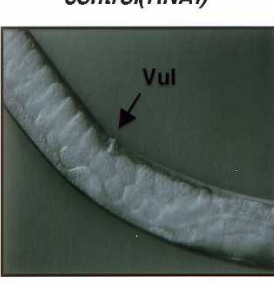

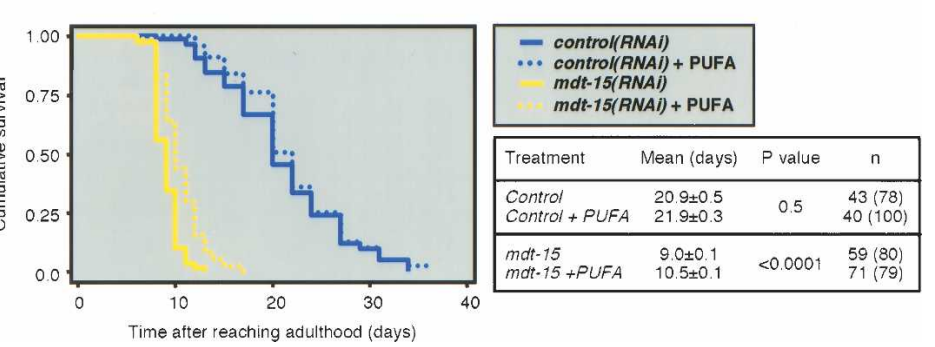

reflect altered expression of non-NHR-49 target genes. We speculated that the reduced PUFA levels may contribute strongly to these pleiotropic phenotypes, as PUFAs have been shown to be important for neurotransmission, development, fertility, and normal behavior in C. elegans (Watts and Browse 2002; Lesa et al. 2003; Kahn-Kirby et al. 2004).

\section{Dietary PUFAs partially suppress the pleiotropic phenotypes observed after reduced MDT-15 expression}

If certain defects exhibited by $m d t-15$ (RNAi) animals indeed result directly from reduced PUFA levels, some of these phenotypes might be complemented by exogenous dietary PUFAs, as seen previously by feeding various unsaturated FAs to fat-3(wa22) mutant animals (KahnKirby et al. 2004). We therefore grew mdt-15(RNAi) worms on plates supplemented with different MUFAs and PUFAs. Whereas weak or no effects were apparent after supplementation with either $200 \mu \mathrm{M}$ C18:2n6, $\mathrm{C} 18: \ln 7$, or C18:1n9, addition of $200 \mu \mathrm{M}$ C20:5 or C20:3n6 resulted in partial suppression of both morphological defects and uncoordinated behavior (data not shown). Moreover, when we grew worms on plates containing a combination of $100 \mu \mathrm{M}$ C20:5 and $100 \mu \mathrm{M}$ C20:3n6, we observed that most mdt-15(RNAi) animals moved normally, occasionally produced viable offspring, 
and exhibited regular gonadal and intestinal morphology (Fig. 6B; data not shown). In these animals, the diffuse fat staining by Nile Red was still present; however, we also detected some fat droplets, which resembled the wild-type fat storage pattern (Fig. 4, right panel). Finally, PUFAs (combination of $100 \mu \mathrm{M}$ C20:5 and $100 \mu \mathrm{M}$ C20:3n6) also partially suppressed the short life-span phenotype of CF512 mdt-15(RNAi) worms (Fig. 6C). Taken together, these results demonstrate that some of the mdt-15 RNAi phenotypes were suppressed at least partially by dietary supplementation with specific FAs.

\section{Discussion}

Precise maintenance of energy homeostasis is critical for all organisms. In this context, it might be expected that the process would be influenced and controlled by multiple components and pathways. Van Gilst et al. (2005a) showed that, in C. elegans, NHR-49 controls two distinct branches of FA metabolism (FA- $\beta$-oxidation and FA- $\Delta$ 9-desaturation). Here we report the identification of the predicted Mediator subunit MDT-15 as an NHR-49 coactivator. In addition, we demonstrate that MDT-15 coregulates a subset of FA-metabolism genes that are not NHR-49 target genes. Our results reveal a previously unrecognized role for MDT-15, and presumably the Mediator complex as a whole, in the regulation of FA metabolism in metazoans. An interaction between orthologous proteins may be relevant to similar regulatory pathways in mammals, as both NHR-49 and MDT-15 are evolutionarily conserved.

\section{MDT-15 is an NHR-49 coregulator in vivo}

NHR-49 regulates FA- $\beta$-oxidation by controlling expression of the acs-2, $1 b p-8$, and cpt-5 genes, and it is necessary for up-regulation of mRNA levels of acs-2, acs-11, gei-7, and hacd-1 in response to short-term fasting; in addition, NHR-49 modulates FA- $\Delta 9$-desaturation by activating expression of fat-5 and fat-7 (Van Gilst et al. 2005a,b). Similarly, knockdown of MDT-15 by RNAi results in defective expression of all these genes in fed and/or fasted worms. Taken together with the initial identification of MDT-15 in a screen for direct physical interaction with the NHR-49-LBD, with the activation capability of MDT-15 in yeast, and with the fact that both proteins are expressed in the intestine, these findings strongly suggest that MDT-15 is a coregulator of NHR-49 in vivo.

MDT-15 is particularly critical for fasting-induced elevation of mRNA levels of acs-2, acs-11, gei-7, and hacd1. It is tempting to speculate that such up-regulation might be achieved through increased association of MDT-15 with the NHR-49-LBD, perhaps driven by binding of the receptor to a hormone-like small molecule(s) whose levels fluctuate in response to food availability. It may be significant in this regard that specific FAs associate with the LBD of HNF4 $\alpha$ (Hertz et al. 1998; Wisely et al. 2002). Thus, nutrient availability may signal to NHR-49:MDT-15 regulatory complexes to modulate a sector of metabolic gene expression.
MDT-15 coordinates expression of $\mathrm{C}$. elegans FA-D9-desaturases

Regardless of nutritional state, MDT-15 is necessary for expression of NHR-49 targets fat-5 and fat-7; in addition, and independently of NHR-49, MDT-15 is required for maintenance of normal fat-6 mRNA levels. Consistent with the extremely low expression of all three FA- $\Delta 9$ desaturases after $m d t-15$ RNAi, these worms exhibited reduced abundance of MUFAs and PUFAs. The coordination of FA- $\Delta$ 9-desaturase expression by a single factor, MDT-15, is compelling, as unsaturated FAs are critical for many aspects of nematode viability (Lesa et al. 2003; Kahn-Kirby et al. 2004); indeed, mdt-15 RNAi severely compromised survival. Dietary supplementation with PUFAs, and particularly a combination of C20:3n 6 and C20:5, partially suppressed the pleiotropic phenotypes associated with mdt-15 RNAi, implicating FA- $\Delta 9$-desaturase activity as a significant contributor to $C$. elegans viability. However, phenotypic suppression was incomplete, perhaps implying poor PUFA bioavailability upon feeding, or roles for additional MDT-15-dependent pathways not explored here.

Expanded target gene specificity of MDT-15 compared with NHR-49

The number of MDT-15-regulated fat-metabolism genes exceeded the number of genes deregulated in nhr49(nr2041) or nhr-49(RNAi) worms, implying that regulatory factors other than NHR-49 use MDT-15 to confer metabolic gene regulation. Candidate factors include several NHRs (NHR-8, NHR-10, NHR-25, NHR-68, NHR-88, NHR-137, NHR-140, NHR-178, and DAF-12) known to affect fat storage in C. elegans (Ashrafi et al. 2003). However, RNAi against these NHRs failed to significantly alter mRNA levels of FA-metabolism genes investigated in this and a previous study (Van Gilst et al. 2005a). Moreover, the LBDs of six of these NHRs (NHR68, NHR-88, NHR-137, NHR-140, NHR-178, and DAF12) did not associate with MDT-15 in our two-hybrid assay (Fig. 1). Other candidates include NHR-64 (which can bind MDT-15 in the two-hybrid assay [this study] and is expressed in the gut, among other tissues [Gissendanner et al. 2004]) and SBP-1, the only C. elegans ortholog of human SREBP proteins (which regulate lipid and cholesterol metabolism and interact with Mediator [Naar et al. 1999; Toth et al. 2004]). In any case, our data indicate that MDT-15 controls the expression of a subset of FA-metabolism genes by interacting with multiple regulatory factors. Clearly, however, not all energy-metabolism genes are affected, as mdt-15 RNAi had only mild effects on the expression of 43 glucose-metabolism genes.

The selectivity of MDT-15 action is reminiscent of the reported effects on gene expression by Mediator subunits MED6 in yeast, MDT-1.1/SOP-3 and MDT-13/LET-19 in C. elegans, and MED23/SUR2 and MED1 in mammals (Zhang and Emmons 2001; Ge et al. 2002; Wang et al. 2004, 2005; Yoda et al. 2005). However, these studies 
focus on the relationship of an individual Mediator subunit with a single target gene or regulator, whereas MDT-15 appears to integrate the actions of multiple regulatory factors that govern a broad physiological process.

MDT-15 may participate in multiple signal transduction cascades

MDT-15-related proteins are conserved throughout fungal and metazoan genomes (Novatchkova and Eisenhaber 2004), but their physiological functions have not been broadly considered. One well-characterized MDT15 ortholog is yeast GAL11, which is a direct target of the regulatory factors GAL4 and GCN4 (Fishburn et al. 2005; Reeves and Hahn 2005); interestingly, $\Delta$ gal11 cells express GCN4 targets normally, but are defective in expression of GAL4 targets. Consistent with this, GCN4 and GAL4 recruit multiple regulatory complexes to achieve activated transcription (Bryant and Ptashne 2003; Govind et al. 2005). It is perhaps significant that both GAL4 and GCN4 regulate transcription of carbohydrate metabolic genes, implying that although MDT-15 lacks a major role in glucose homeostasis, it may be involved in other aspects of carbohydrate metabolism. In any case, the fact that two orthologous proteins govern metabolic responses is striking, and indicates that transcriptional control of energy homeostasis may represent an evolutionarily ancient function of Mediator.

Another MDT-15 ortholog, Xenopus laevis XARC105, induces neural and mesendodermal genes specifically in response to activin/TGF- $\beta$ signaling (Kato et al. 2002). As the Smad/TGF- $\beta$ pathway is well conserved in $C$. elegans, MDT-15 may therefore participate in TGF- $\beta$ signal transduction in C. elegans. Alternatively, Smad: MDT-15 complexes may also be important for regulation of NHR-49-independent FA-metabolism genes. Clearly, it will be interesting in future studies to identify additional MDT-15 targets in C. elegans using unbiased approaches such as expression microarrays.

\section{MDT-15 is a predicted subunit of the tail domain of Mediator}

Structural models of the Mediator complex suggest that MDT-15 orthologs reside in the so-called tail domain (Asturias et al. 1999; Guglielmi et al. 2004), which may directly contact regulatory factors. Curiously, the genome of C. elegans contains predicted orthologs of only two of the five subunits found in the yeast tail domain, MDT-15 and MDT-14/RGR-1. Conceivably, one or more of the metazoan-specific Mediator subunits (MDT-23/ SUR-2, MDT-28, and MDT-29) could substitute for the absent MED2/3/16 subunits. Alternatively, MDT-14/ RGR-1 and/or MDT-15 might contribute additional interactions in C. elegans, such as association with factors like NHRs, which are absent from fungal genomes. Another possibility is that the C. elegans Mediator tail domain simply contains additional unidentified proteins.
Although multiple Mediator subunits have been found to directly interact with NHRs (Blazek et al. 2005), this is the first report describing an interaction of an NHR with MDT-15/MED15 proteins. NHRs commonly target coregulators via canonical LxxLL motifs. However, no such motif is present in the predicted MDT-15 ORF; instead, it appears that the GACKIX domain constitutes the target for NHR-49. It is noteworthy that there are structural similarities between the interfaces of LxxLL motifs and GACKIX domains and their respective targets (Radhakrishnan et al. 1997). Thus, it is tempting to speculate that KIX-related domains in human MED15 and, potentially, p300/CBP represent non-LxxLL interaction surfaces for human NHRs as well.

\section{Similarities and differences between MDT-15 and mammalian PGC-1}

In general, transcriptional regulators serve as nucleation centers for the assembly of multiprotein complexes that include numerous factors in addition to Mediator. In the case of the mammalian NHRs PPAR $\alpha$ and $\mathrm{HNF} 4 \alpha$, PGC-1 proteins serve as coactivators that control several distinct pathways involved in energy homeostasis (Puigserver and Spiegelman 2003; Lin et al. 2005). For example, in response to short-term fasting, PGC-1 $\alpha$ coactivates PPAR $\alpha$ and $\mathrm{HNF} 4 \alpha$ to induce gluconeogenesis and $\beta$-oxidation in liver, adipose, and heart (Yoon et al. 2001; Rhee et al. 2003). Similarly, MED1 is required for ligand-stimulated PPAR $\alpha$-dependent gene expression in the liver (Jia et al. 2004), and for PPAR $\gamma$-dependent adipocyte differentiation (Ge et al. 2002). Thus, these two coregulators govern metabolic and cell fate decisions by integrating hormonal signals and nutrient state.

Curiously, the genome of C. elegans appears to lack orthologs of the PGC-1 proteins (Knutti et al. 2000). However, our data reveal partial functional overlap between NHR-49:MDT-15 and PPAR $\alpha$ :PGC-1 and/or HNF4 $\alpha$ :PGC-1. Specifically, both the C. elegans and the mammalian factor pairs appear to collaborate to regulate FA- $\beta$-oxidation and FA- $\Delta 9$-desaturation. In contrast to the mammalian proteins, however, NHR-49:MDT-15 fails to strongly modulate glucose-metabolism genes. In any case, MDT-15 appears to orchestrate many of the principal metabolic changes that occur in C. elegans in response to short-term fasting.

Given that mammalian MED1, and thus Mediator, play prominent roles in adipocyte cell fate specification and differentiation, it would seem worthwhile to investigate the contributions of MED15 in these metabolic processes in mammalian model systems. In particular, in view of our findings in C. elegans, it will be interesting to determine whether mammalian MED15 proteins are involved in regulation of FA- $\beta$-oxidation genes in response to short-term fasting. Our results suggest that MDT-15, at least in C. elegans, integrates distinct hormonal and nutritional pathways governing fat homeostasis, thereby assuring proper energy balance. We speculate that, through distinct metabolic regulatory factors, MDT-15 orthologs play similar roles in mammals, and 
thus may be important in conditions such as metabolic syndrome and/or diabetes.

\section{Materials and methods}

\section{Yeast two-hybrid screen}

The LBDs of NHR-3, NHR-14, NHR-31, NHR-35, NHR-49, NHR-64, NHR-68, NHR-88, NHR-137, NHR-140, NHR-178, and DAF-12, and the GACKIX and NT domains of MDT-15 were amplified by PCR using Pfu Turbo DNA polymerase (Stratagene \#600250) and mixed-stage C. elegans cDNA as template (primer sequences available upon request). The resulting cDNAs were sequenced and subcloned into the vector pGBKLeu2, which carries a Leu2 auxotrophy marker (instead of Trp of the parental pGBKT7; Clontech), to generate plasmids expressing individual GAL4-DBD-NHR-LBD fusions.

Plasmids were transformed into yeast strain AH109 (Clontech) using standard PEG3350/LiAc methods. Expression of fusion proteins was verified by standard lysis, SDS-PAGE, and Western blot techniques (Santa Cruz sc-40 anti-Myc [9E10] monoclonal antibody). For the NHR-49-LBD yeast two-hybrid screen, strain AH109/pGBK-Leu2-NHR-49-LBD was used to probe a C. elegans oligo(dT)-primed cDNA library. We screened $\sim 4.3 \times 10^{6}$ independent transformants for growth on minimal medium lacking tryptophan, leucine, histidine, and uracil containing 7.5 mM 3-amino-1,2,4-triazole (3-AT; Sigma, H-8056), and recovered 142 interacting clones, yielding 23 independent cDNAs of 12 genes. Six of the clones harbored the identical full-length cDNA corresponding to R12B2.5 (mdt-15). The cDNA was fused in frame to the GAL4-AD, and contained $90 \mathrm{nt}$ of $5^{\prime}$ untranslated region, resulting in a 30 -amino-acid linker between the GAL4-AD and the ATG of the MDT-15 ORF (data not shown). To estimate relative interaction strength, we transformed plasmid pairs into strain Y187 (Clontech) and performed liquid $\beta$-galactosidase assays as described in the manufacturer's protocol.

For the MDT-15-GACKIX yeast two-hybrid screen, we probed the same library using identical conditions. We screened $\sim 3.5 \times 10^{6}$ independent clones, and recovered two independent plasmids encoding NHR-49B, both of which were in-frame fusions.

\section{Nematode strains and growth conditions}

C. elegans strains N2-Bristol (wild type [WT]), nhr-49(nr2041) (Van Gilst et al. 2005a), CF512 [fer-15(b26)II; fem-1(hc17)III)] (Garigan et al. 2002), and BC11928 [dpy-5(e907)/dpy-5(e907); sIs10941 (rCesR12B2.5a::GFP + pCeh361)] (S.J. McKay et al. 2003) were maintained as described (Van Gilst et al. 2005a). For mRNA and GC/MS analysis, worm embryos were allowed to hatch on unseeded nematode growth media (NGM)-lite plates overnight at $20^{\circ} \mathrm{C}$. The next day, synchronized L1 larvae were plated onto NGM-lite-RNAi plates (NGM-lite containing 25 $\mu \mathrm{g} / \mathrm{mL}$ Carbenicillin, $4 \mathrm{mM}$ IPTG [Alexis 582-600], and 12.5 $\mu \mathrm{g} / \mathrm{mL}$ tetracyclin) seeded with Escherichia coli strain HT115 carrying the appropriate RNAi vector. Worms were grown to early L4s at $20^{\circ} \mathrm{C}$ ( $34 \mathrm{~h}$ growth), harvested, washed twice with M9, and replated on RNAi plates (fed condition) or unseeded NGM-lite plates (fasted condition). After $8 \mathrm{~h}$, worms were harvested, washed with $\mathrm{M} 9$, and flash-frozen in liquid $\mathrm{N}_{2}$. For adults, CF512 worms were grown at $16^{\circ} \mathrm{C}$ (permissive temperature), but embryos were hatched overnight at $25^{\circ} \mathrm{C}$ (restrictive temperature). Synchronized L1 larvae were collected, plated onto NGM-lite plates seeded with OP50, and grown to young adults at $25^{\circ} \mathrm{C}$. Subsequently, they were grown on RNAi plates for $34 \mathrm{~h}$, and then fed or fasted as described.

\section{RNAi constructs}

The mdt-15 RNAi construct (from the Ahringer RNAi library) (Kamath et al. 2003) was sequenced to confirm its identity. The sequence of the insert was subjected to BLAST search, which confirmed that there was no other sequence of significant homology in the C. elegans genome (data not shown). Moreover, an $m d t-15$ RNAi clone of the Vidal RNAi library (Rual et al. 2004) similarly affected relative mRNA levels of MDT-15 targets (data not shown). The mdt-6 construct was also from the Ahringer library; the nhr-49 clone has been described (Van Gilst et al. 2005a).

Preparation of total nematode mRNA, qRT-PCR analysis, and $G C / M S$ analysis

Isolation, purification, and reverse transcription of C. elegans RNA, as well as isolation of FAs and GC/MS analysis were carried out as described (Van Gilst et al. 2005a,b). QRT-PCR was performed in an Opticon 2 DNA Engine (MJ Research), analyzed using the $\mathrm{C}_{\mathrm{t}}$ method (Applied Biosystems Prism 7700 Users Bulletin No. 2; http://docs.appliedbiosystems.com/pebiodocs/ 04303859.pdf), and normalized to ama-1. Primers for qRT-PCR analysis have been described (Shostak et al. 2004; Van Gilst et al. $2005 \mathrm{a}, \mathrm{b})$ and are available upon request.

\section{Life-span analysis}

Life-span analysis was conducted as described (Hansen et al. 2005). The prefertile period of adulthood was used as $t=0$ for life-span analysis. STATA 9 software was used for statistical analysis. In all cases, $P$ values were calculated using the Logrank (Mantel-Cox) method.

\section{DIC and fluorescence microscopy}

Worms were grown on NGM-lite RNAi plates seeded with $E$. coli strain HT115 carrying the appropriate RNAi vector; plates contained Nile Red (50 ng/mL; N-1142; Molecular Probes) as required. Worms were transferred onto $2 \%(\mathrm{w} / \mathrm{v})$ agarose pads for microscopic examination. Images were captured with a Zeiss Axioplan II microscope equipped with rhodamine (emission 560-590 nm) and FITC/GFP (emission 500-515 nm) filters. All Nile Red images were acquired using identical settings and exposure times.

\section{PUFA complementation analysis}

Stocks of unsaturated FAs (Nu-Chek-Prep, Inc.) were prepared by dissolving FA-sodium-salts to $100 \mathrm{mM}$ in $\mathrm{ddH}_{2} \mathrm{O}$ immediately prior to pouring plates. NGM-lite was autoclaved and cooled to $50^{\circ} \mathrm{C}$, and FAs were added to final concentrations of $100 \mu \mathrm{M}$ or $200 \mu \mathrm{M}$, respectively. Plates were dried in the dark at room temperature, and seeded with HT115 bacteria carrying the appropriate RNAi vector.

\section{Acknowledgments}

We thank M. Vidal and D. Hill for the cDNA library, and the laboratories of D. Baillie and D. Moerman for BC11928 worms (strain construction supported by the Genome British Columbia and Genome Canada). We are grateful to J. Ahringer for RNAi 
vectors, and C. Kenyon for the use of microscopes. We also thank R.V. Farese Jr., K. Ashrafi, G. Brown, C. Pantoja, and J.-C. Wang for critical comments on the manuscript, and members of the Yamamoto lab for discussions. S.T. was partially supported by an AHA Post-doctoral Fellowship, M.R.V.G. by a NIH grant (NIH K01 DK61361-01), and M.H. by an Ellison/AFAR Senior Post-doctoral Fellowship. Research support was from the NIH (to K.R.Y.).

\section{References}

Ashrafi, K., Chang, F.Y., Watts, J.L., Fraser, A.G., Kamath, R.S., Ahringer, J., and Ruvkun, G. 2003. Genome-wide RNAi analysis of Caenorhabditis elegans fat regulatory genes. $\mathrm{Na}$ ture 421: 268-272.

Asturias, F.J., Jiang, Y.W., Myers, L.C., Gustafsson, C.M., and Kornberg, R.D. 1999. Conserved structures of mediator and RNA polymerase II holoenzyme. Science 283: 985-987.

Blazek, E., Mittler, G., and Meisterernst, M. 2005. The mediator of RNA polymerase II. Chromosoma 113: 399-408.

Bourbon, H.M., Aguilera, A., Ansari, A.Z., Asturias, F.J., Berk, A.J., Bjorklund, S., Blackwell, T.K., Borggrefe, T., Carey, M., Carlson, M., et al. 2004. A unified nomenclature for protein subunits of mediator complexes linking transcriptional regulators to RNA polymerase II. Mol. Cell 14: 553-557.

Bryant, G.O. and Ptashne, M. 2003. Independent recruitment in vivo by Gal4 of two complexes required for transcription. Mol. Cell 11: 1301-1309.

Desvergne, B. and Wahli, W. 1999. Peroxisome proliferator-activated receptors: Nuclear control of metabolism. Endocr. Rev. 20: 649-688.

Dowell, P., Ishmael, J.E., Avram, D., Peterson, V.J., Nevrivy, D.J., and Leid, M. 1997. p300 functions as a coactivator for the peroxisome proliferator-activated receptor $\alpha$. J. Biol. Chem. 272: 33435-33443.

. 1999. Identification of nuclear receptor corepressor as a peroxisome proliferator-activated receptor $\alpha$ interacting protein. J. Biol. Chem. 274: 15901-15907.

Evans, R.M. 1988. The steroid and thyroid hormone receptor superfamily. Science 240: 889-895.

Fishburn, J., Mohibullah, N., and Hahn, S. 2005. Function of a eukaryotic transcription activator during the transcription cycle. Mol. Cell 18: 369-378.

Garigan, D., Hsu, A.L., Fraser, A.G., Kamath, R.S., Ahringer, J., and Kenyon, C. 2002. Genetic analysis of tissue aging in Caenorhabditis elegans: A role for heat-shock factor and bacterial proliferation. Genetics 161: 1101-1112.

Garofalo, A., Rowlinson, M.C., Amambua, N.A., Hughes, J.M., Kelly, S.M., Price, N.C., Cooper, A., Watson, D.G., Kennedy, M.W., and Bradley, J.E. 2003. The FAR protein family of the nematode Caenorhabditis elegans. Differential lipid binding properties, structural characteristics, and developmental regulation. J. Biol. Chem. 278: 8065-8074.

Ge, K., Guermah, M., Yuan, C.X., Ito, M., Wallberg, A.E., Spiegelman, B.M., and Roeder, R.G. 2002. Transcription coactivator TRAP220 is required for PPAR $\gamma 2$-stimulated adipogenesis. Nature 417: 563-567.

Gissendanner, C.R., Crossgrove, K., Kraus, K.A., Maina, C.V., and Sluder, A.E. 2004. Expression and function of conserved nuclear receptor genes in Caenorhabditis elegans. Dev. Biol. 266: 399-416.

Glass, C.K. and Rosenfeld, M.G. 2000. The coregulator exchange in transcriptional functions of nuclear receptors. Genes \& Dev. 14: 121-141.

Govind, C.K., Yoon, S., Qiu, H., Govind, S., and Hinnebusch,
A.G. 2005. Simultaneous recruitment of coactivators by Gen $4 p$ stimulates multiple steps of transcription in vivo. Mol. Cell. Biol. 25: 5626-5638.

Guglielmi, B., van Berkum, N.L., Klapholz, B., Bijma, T., Boube, M., Boschiero, C., Bourbon, H.M., Holstege, F.C., and Werner, M. 2004. A high resolution protein interaction map of the yeast Mediator complex. Nucleic Acids Res. 32: 53795391.

Handschin, C. and Meyer, U.A. 2005. Regulatory network of lipid-sensing nuclear receptors: Roles for CAR, PXR, LXR, and FXR. Arch. Biochem. Biophys. 433: 387-396.

Hansen, M., Hsu, A.L., Dillin, A., and Kenyon, C. 2005. New genes tied to endocrine, metabolic, and dietary regulation of lifespan from a Caenorhabditis elegans genomic RNAi screen. PLoS Genet. 1: 119-128.

Hertz, R., Magenheim, J., Berman, I., and Bar-Tana, J. 1998. Fatty acyl-CoA thioesters are ligands of hepatic nuclear factor-4 $\alpha$. Nature 392: 512-516.

Jia, Y., Qi, C., Kashireddi, P., Surapureddi, S., Zhu, Y.J., Rao, M.S., Le Roith, D., Chambon, P., Gonzalez, F.J., and Reddy, J.K. 2004. Transcription coactivator PBP, the peroxisome proliferator-activated receptor (PPAR)-binding protein, is required for PPAR $\alpha$-regulated gene expression in liver. J. Biol. Chem. 279: 24427-24434.

Kahn-Kirby, A.H., Dantzker, J.L., Apicella, A.J., Schafer, W.R., Browse, J., Bargmann, C.I., and Watts, J.L. 2004. Specific polyunsaturated fatty acids drive TRPV-dependent sensory signaling in vivo. Cell 119: 889-900.

Kamath, R.S., Fraser, A.G., Dong, Y., Poulin, G., Durbin, R., Gotta, M., Kanapin, A., Le Bot, N., Moreno, S., Sohrmann, M., et al. 2003. Systematic functional analysis of the Caenorhabditis elegans genome using RNAi. Nature 421: 231237.

Kato, Y., Habas, R., Katsuyama, Y., Naar, A.M., and He, X. 2002 A component of the ARC/Mediator complex required for TGF $\beta /$ Nodal signalling. Nature 418: 641-646.

Kimura, K.D., Tissenbaum, H.A., Liu, Y., and Ruvkun, G. 1997. daf-2, an insulin receptor-like gene that regulates longevity and diapause in Caenorhabditis elegans. Science 277: 942 946.

Knutti, D., Kaul, A., and Kralli, A. 2000. A tissue-specific coactivator of steroid receptors, identified in a functional genetic screen. Mol. Cell. Biol. 20: 2411-2422.

Kwon, J.Y. and Lee, J. 2001. Biological significance of a universally conserved transcription mediator in metazoan developmental signaling pathways. Development 128: 3095-3104.

Lee, S.S., Pineau, T., Drago, J., Lee, E.J., Owens, J.W., Kroetz, D.L., Fernandez-Salguero, P.M., Westphal, H., and Gonzalez, F.J. 1995. Targeted disruption of the $\alpha$ isoform of the peroxisome proliferator-activated receptor gene in mice results in abolishment of the pleiotropic effects of peroxisome proliferators. Mol. Cell. Biol. 15: 3012-3022.

Lesa, G.M., Palfreyman, M., Hall, D.H., Clandinin, M.T., Rudolph, C., Jorgensen, E.M., and Schiavo, G. 2003. Long chain polyunsaturated fatty acids are required for efficient neurotransmission in C. elegans. J. Cell Sci. 116: 4965-4975.

Lin, J., Handschin, C., and Spiegelman, B.M. 2005. Metabolic control through the PGC-1 family of transcription coactivators. Cell Metab. 1: 361-370.

Ludewig, A.H., Kober-Eisermann, C., Weitzel, C., Bethke, A., Neubert, K., Gerisch, B., Hutter, H., and Antebi, A. 2004. A novel nuclear receptor/coregulator complex controls C. elegans lipid metabolism, larval development, and aging. Genes \& Dev. 18: 2120-2133.

Malik, S. and Roeder, R.G. 2005. Dynamic regulation of Pol II transcription by the mammalian Mediator complex. Trends 
Biochem. Sci. 30: 256-263.

Malik, S., Guermah, M., Yuan, C.X., Wu, W., Yamamura, S., and Roeder, R.G. 2004. Structural and functional organization of TRAP220, the TRAP/mediator subunit that is targeted by nuclear receptors. Mol. Cell. Biol. 24: 8244-8254.

McKay, R.M., McKay, J.P., Avery, L., and Graff, J.M. 2003. C. elegans: A model for exploring the genetics of fat storage. Dev. Cell 4: 131-142.

McKay, S.J., Johnsen, R., Khattra, J., Asano, J., Baillie, D.L., Chan, S., Dube, N., Fang, L., Goszczynski, B., Ha, E., et al. 2003. Gene expression profiling of cells, tissues, and developmental stages of the nematode C. elegans. Cold Spring Harb. Symp. Quant. Biol. 68: 159-169.

Naar, A.M., Beaurang, P.A., Zhou, S., Abraham, S., Solomon, W., and Tjian, R. 1999. Composite co-activator ARC mediates chromatin-directed transcriptional activation. Nature 398: 828-832.

Nakamura, M.T., Cheon, Y., Li, Y., and Nara, T.Y. 2004. Mechanisms of regulation of gene expression by fatty acids. Lipids 39: $1077-1083$

Novatchkova, M. and Eisenhaber, F. 2004. Linking transcriptional mediators via the GACKIX domain super family. Curr. Biol. 14: R54-R55.

Puigserver, P. and Spiegelman, B.M. 2003. Peroxisome proliferator-activated receptor $\gamma$ coactivator $1 \propto($ PGC- $1 \alpha)$ : Transcriptional coactivator and metabolic regulator. Endocr. Rev. 24: 78-90.

Radhakrishnan, I., Perez-Alvarado, G.C., Parker, D., Dyson, H.J., Montminy, M.R., and Wright, P.E. 1997. Solution structure of the KIX domain of CBP bound to the transactivation domain of CREB: A model for activator:coactivator interactions. Cell 91: 741-752.

Reeves, W.M. and Hahn, S. 2005. Targets of the Gal4 transcription activator in functional transcription complexes. Mol. Cell. Biol. 25: 9092-9102.

Rhee, J., Inoue, Y., Yoon, J.C., Puigserver, P., Fan, M., Gonzalez, F.J., and Spiegelman, B.M. 2003. Regulation of hepatic fasting response by PPAR $\gamma$ coactivator- $1 \alpha$ (PGC-1): Requirement for hepatocyte nuclear factor $4 \alpha$ in gluconeogenesis. Proc. Natl. Acad. Sci. 100: 4012-4017.

Rual, J.F., Ceron, J., Koreth, J., Hao, T., Nicot, A.S., HirozaneKishikawa, T., Vandenhaute, J., Orkin, S.H., Hill, D.E., van den Heuvel, S., et al. 2004. Toward improving Caenorhabditis elegans phenome mapping with an ORFeome-based RNAi library. Genome Res. 14: 2162-2168.

Shostak, Y., Van Gilst, M.R., Antebi, A., and Yamamoto, K.R. 2004. Identification of C. elegans DAF-12-binding sites, response elements, and target genes. Genes \& Dev. 18: 25292544.

Sluder, A.E. and Maina, C.V. 2001. Nuclear receptors in nematodes: Themes and variations. Trends Genet. 17: 206-213.

Sze, J.Y., Victor, M., Loer, C., Shi, Y., and Ruvkun, G. 2000. Food and metabolic signalling defects in a Caenorhabditis elegans serotonin-synthesis mutant. Nature 403: 560-564.

Toth, J.I., Datta, S., Athanikar, J.N., Freedman, L.P., and Osborne, T.F. 2004. Selective coactivator interactions in gene activation by SREBP-1a and -1c. Mol. Cell. Biol. 24: 82888300.

Van Gilst, M., Gissendanner, C.R., and Sluder, A.E. 2002. Diversity and function of orphan nuclear receptors in nematodes. Crit. Rev. Eukaryot. Gene Expr. 12: 65-88.

Van Gilst, M.R., Hadjivassiliou, H., Jolly, A., and Yamamoto, K.R. 2005a. Nuclear hormone receptor NHR-49 controls fat consumption and fatty acid composition in C. elegans. PLoS Biol. 3: e53.

Van Gilst, M.R., Hadjivassiliou, H., and Yamamoto, K.R. 2005b.
A Caenorhabditis elegans nutrient response system partially dependent on nuclear receptor NHR-49. Proc. Nat1. Acad. Sci. 102: 13496-13501.

Vega, R.B., Huss, J.M., and Kelly, D.P. 2000. The coactivator PGC-1 cooperates with peroxisome proliferator-activated receptor $\alpha$ in transcriptional control of nuclear genes encoding mitochondrial fatty acid oxidation enzymes. Mol. Cell. Biol. 20: $1868-1876$.

Wang, J. and Barr, M.M. 2005. RNA interference in Caenorhabditis elegans. Methods Enzymol. 392: 36-55.

Wang, J.C., Walker, A., Blackwell, T.K., and Yamamoto, K.R. 2004. The Caenorhabditis elegans ortholog of TRAP240, CeTRAP240/let-19, selectively modulates gene expression and is essential for embryogenesis. J. Biol. Chem. 279: 29270-29277.

Wang, G., Balamotis, M.A., Stevens, J.L., Yamaguchi, Y., Handa, H., and Berk, A.J. 2005. Mediator requirement for both recruitment and postrecruitment steps in transcription initiation. Mol. Cell 17: 683-694.

Watts, J.L. and Browse, J. 2002. Genetic dissection of polyunsaturated fatty acid synthesis in Caenorhabditis elegans. Proc. Natl. Acad. Sci. 99: 5854-5859.

Wisely, G.B., Miller, A.B., Davis, R.G., Thornquest Jr., A.D., Johnson, R., Spitzer, T., Sefler, A., Shearer, B., Moore, J.T., Miller, A.B., et al. 2002. Hepatocyte nuclear factor 4 is a transcription factor that constitutively binds fatty acids. Structure 10: 1225-1234.

Yoda, A., Kouike, H., Okano, H., and Sawa, H. 2005. Components of the transcriptional Mediator complex are required for asymmetric cell division in C. elegans. Development 132: $1885-1893$.

Yoon, J.C., Puigserver, P., Chen, G., Donovan, J., Wu, Z., Rhee, J., Adelmant, G., Stafford, J., Kahn, C.R., Granner, D.K., et al. 2001. Control of hepatic gluconeogenesis through the transcriptional coactivator PGC-1. Nature 413: 131-138.

Zhang, H. and Emmons, S.W. 2001. The novel C. elegans gene sop-3 modulates Wnt signaling to regulate Hox gene expression. Development 128: 767-777. 


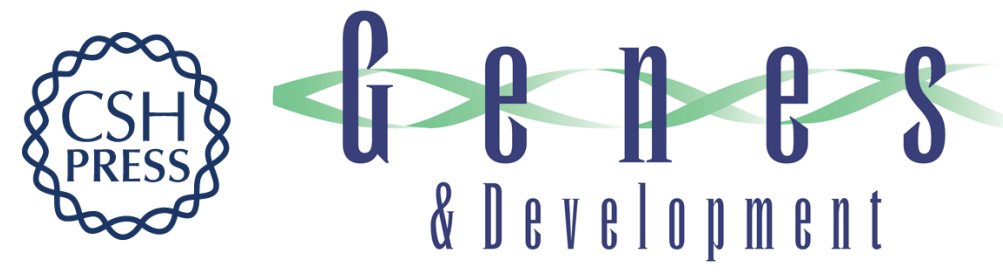

\section{A Mediator subunit, MDT-15, integrates regulation of fatty acid metabolism by NHR-49-dependent and -independent pathways in $C$. elegans}

Stefan Taubert, Marc R. Van Gilst, Malene Hansen, et al.

Genes Dev. 2006, 20:

Access the most recent version at doi:10.1101/gad.1395406

Supplemental http://genesdev.cshlp.org/content/suppl/2006/04/17/20.9.1137.DC1

Material

References This article cites 57 articles, 25 of which can be accessed free at: http://genesdev.cshlp.org/content/20/9/1137.full.html\#ref-list-1

License

Email Alerting Receive free email alerts when new articles cite this article - sign up in the box at the top Service right corner of the article or click here.

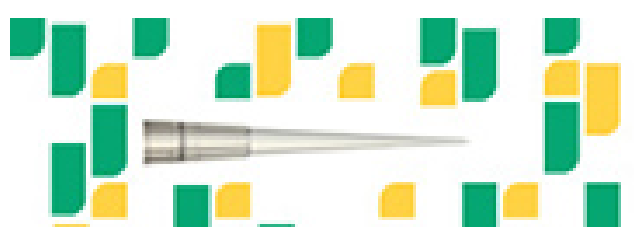

Focused on your science. 